\title{
AVALIAÇÃO DO EFEITO DO ESTRESSE HÍDRICO NO CRESCIMENTO DE CULTIVARES DE CANA-DE-AÇÚCAR USANDO UM SISTEMA AUTOMÁTICO DE FERTIRRIGAÇÃO ${ }^{1}$
}

Doi:http://dx.doi.org/10.1590/1809-4430-Eng.Agric.v35n2p215-229/2015

\section{EVANDRO L. DA S. BATISTA ${ }^{2}$, SÉRGIO ZOLNIER ${ }^{3}$, ARISTIDES RIBEIRO ${ }^{4}$, GUSTAVO B. LYRA ${ }^{5}$, THIERES G. F. DA SILVA ${ }^{6}$, DAVI BOEHRINGER}

\begin{abstract}
RESUMO: O objetivo desta pesquisa foi identificar o efeito de diferentes níveis de estresse hídrico no crescimento das cultivares RB92579, RB855453, RB867515 e RB928064, utilizando um sistema automático de controle dos eventos de fertirrigação, durante o período de formação da cana-de-açúcar. No período experimental, as plantas foram submetidas a períodos alternados de estresse e de reposição hídrica, por meio de fertirrigações periódicas, cujos limites do potencial matricial de água $(\Psi)$ foram aqui denominados, para propósitos comparativos, como ausência de estresse, estresse leve, moderado e severo, respectivamente, para os valores críticos de $\Psi$ de $-10,-60,-90$ e $-120 \mathrm{kPa}$. Para avaliação do efeito do estresse hídrico no crescimento da cana-de-açúcar, foi utilizado o modelo sigmoidal com três parâmetros, tendo graus-dia acumulados como variável independente, o qual demonstrou excelente ajuste aos dados de estatura dos colmos $\left(\mathrm{R}^{2}{ }_{\mathrm{aj}}>0,95\right)$. Na ausência de estresse hídrico, os valores máximos da taxa de elongação dos colmos (TEC) estiveram compreendidos entre 0,23 e $0,26 \mathrm{~cm}^{\circ} \mathrm{Cd}^{-1}$, independentemente da cultivar. Por outro lado, sob estresse severo, os valores máximos da TEC estiveram entre 0,07 e $0,09 \mathrm{~cm}^{\circ} \mathrm{Cd}^{-1}$, também independentemente da cultivar a valiada.
\end{abstract}

PALAVRAS-CHAVE: modelos de crescimento, Saccharum officinarum, disponibilidade hídrica, irrigação.

\section{EVALUATION OF THE WATER STRESS EFFECT ON THE GROWTH OF SUGAR CANE CULTIVARS USING AN AUTOMATIC FER TIGATION SYSTEM}

\begin{abstract}
The objective of this research was to identify the effect of different water stress levels on crop growth of RB92579, RB855453, RB867515 and RB928064 cultivars, by using an automatic system to control fertigation events, during the sugarcane formative phase. In the experimental period, plants were subjected to alternate periods of stress and water supply, by means of periodic fertigations, whose limits of the water matric potential $(\Psi)$ were denoted, for comparison purposes, as absence of water stress and mild, moderate and severe water stress, respectively for critical $\Psi$ values of $-10,-60,-90$ and $-120 \mathrm{kPa}$. For evaluation of the water stress effect on the sugarcane growth, it was utilized the sigmoid model with three parameters and degree-days as independent variable, which demonstrated excellent adjust to stalk length $\left(\mathrm{R}_{\mathrm{aj}}^{2}>0.95\right)$. Under the absence of water stress, the maximum values of the stalk elongation rate (SER) ranged from 0.23 to $0.26 \mathrm{~cm}^{\circ} \mathrm{Cd}^{-1}$, regardless of the cultivar. In contrast, under severe water stress, the maximum SER values were between 0.07 and $0.09 \mathrm{~cm}^{\circ} \mathrm{Cd}^{-1}$, also regardless of the evaluated cultivar.
\end{abstract}

KEYWORDS: growth models, Saccharum officinarum, water availability, irrigation.

\footnotetext{
${ }^{1}$ Extraído da Dis sertação de Mestrado do primeiro autor. Bols a de estudos concedida pelo CNPq e au xílio financeiro para execução do projeto de pesquis a proveniente da FAPEMIG e do CNPq.

${ }^{2} \mathrm{Eng}^{\mathrm{o}}$ Agrônomo, Doutorando em Meteorologia Agrícola, Departamento de Engenharia Agrícola, DEA/UFV,

Viçosa-MG, 36571-000, evandrolsb@yahoo.com.br

${ }^{3}$ Eng $^{\circ}$ Agrícola, Prof. Doutor, Departamento de Engenharia Agrícola, UFV, Viçosa - MG, zolnier@ufv.br

${ }^{4}$ Eng $^{\circ}$ Agrônomo, Prof. Doutor, Departamento de Engenharia Agrícola, UFV, Viçosa - MG, ribeiro.ufv@g mail.com

${ }^{5}$ Meteorologista, Prof. Doutor, Departamento de Ciências Ambientais, UFRRJ, Rio de Janeiro - RJ, gblyra@ @frrj.br

${ }^{6}$ Eng ${ }^{\text {o }}$ Agrônomo, Prof. Doutor, Unidade Acadêmica de Serra Talhada, UFRPE, Serra Talhada - PE, thigeoprofissional@hotmail.com

${ }^{7}$ Eng $^{\circ}$ Agrônomo, Doutorando em Meteorologia Agrícola, Departamento de Engenharia Agrícola, DEA/UFV,

Viçosa-MG, davib82@hotmail.com
}

Recebido pelo Conselho Editorial em: 17-2-2014

Aprovado pelo Conselho Editorial em: 19-9-2014 


\section{INTRODUÇÃO}

A área cultivada com cana-de-açúcar, referente à safra de 2013/2014, deve alcançar aproximadamente 8,81 milhões de hectares, com produção estimada de 659,85 milhões de toneladas, o que corresponde ao aumento de 12,0\% quando comparada com a produção de 2012/2013 (CONAB, 2013).

Uma vez que a maior parte do cultivo de cana-de-açúcar ocorre em regime de sequeiro no Brasil, a cultura está sujeita à ocorrência de déficit hídrico em determinadas fases de seu desenvolvimento ou mesmo durante todo o ciclo (LYRA et al., 2012). Isso ocorre devido à irregular distribuição sazonal das chuvas nas regiões produtoras e à ocorrência de veranicos, que frequentemente ocorrem no período chuvoso (SILVA et al., 2013). Sob condições de cultivo em sequeiro, estudos sobre o consumo de água da cana-de-açúcar tornam-se importantes em razão de existir uma variação genotípica de resposta ao estresse hídrico por diferentes variedades (TRENTIN et al., 2011; BATISTA et al., 2013; BOEHRINGER et al., 2013).

Durante o ciclo de crescimento, as fases de perfilhamento e de crescimento intenso constituem a fase de formação da cultura (RAMESH, 2000). Esse é o período mais crítico no que se refere aos efeitos do déficit hídrico na produtividade, pois é quando as plantas apresentam grande área foliar e necessitam de maior quantidade de água para a realização das trocas gasosas com a atmosfera (GONÇALVES et al., 2010; SILVA et al., 2011a; TRENTIN et al., 2011). Nesta fase, a ocorrência do estresse hídrico acarreta efeitos mais severos na redução das taxas de crescimento do colmo, na produção de fitomassa e no rendimento de sacarose, cujas respostas serão diferentes entre as cultivares (MACHADO et al., 2009; GONÇALVES et al., 2010; GAVA et al., 2011; SILVA et al., 2011b).

A identificação dos padrões de crescimento possibilita selecionar cultivares adaptadas aos diferentes ambientes de produção e que sejam eficientes no uso da água (OLIVEIRA et al., 2010). Fundamentada na medida sequencial do acúmulo de biomassa e de variáveis agronômicas e biofísicas, tais como produção de matéria seca e verde, altura da planta e comprimento das folhas, a análise de crescimento permite o cálculo de alguns índices, tais como taxa de crescimento da cultura e taxa de crescimento relativo, sendo então possível a estimativa das curvas de acúmulo de biomassa em componentes estruturais e do acúmulo total ao longo do ciclo da cultura (OLIVEIRA et al., 2005; SILVA et al., 2009; SILVA et al., 2012; NOBILE et al., 2011).

No caso específico da cana-de-açúcar, estudos de análise de crescimento têm sido conduzidos, em países onde é extensivamente cultivada, a fim de melhorar o entendimento da influência dos elementos meteorológicos e do estresse hídrico no rendimento final. Estes estudos podem auxiliar a seleção de cultivares mais adaptadas a ambientes de produção específicos (OLIVEIRA et al., 2010; ZHAO et al., 2010; OLIVEIRA et al., 2011).

Em decorrência das características de crescimento do sistema radicular da cana-de-açúcar em condições de campo, o qual pode ultrapassar 4 m (LACLAU \& LACLAU, 2009) e até mesmo 6 m de profundidade (SMITH et al., 2005), a quantidade de água armazenada no solo e disponível para as plantas sofre a influência de vários fatores, principalmente compactação, estrutura e textura do solo. Além desses fatores, o conhecimento da disponibilidade de água em estudos de estresse hídrico em condições de campo é limitado, uma vez que determinada cultivar, submetida a um tratamento específico, a partir da medição do potencial matricial nas camadas superficiais, pode utilizar parte da água armazenada nos níveis inferiores do solo, dificultando, assim, a interpretação da tolerância ao estresse hídrico pelas diversas cultivares avaliadas.

Em casa de vegetação, sistemas automáticos de controle dos eventos de aplicação de água e do preparo de soluções nutritivas para implementação da fertirrigação têm sido propostos por diversos pesquisadores, com o objetivo de avaliar a resposta dinâmica destes sistemas diante de alterações das condições meteoroló gicas do ambiente de cultivo (DOMINGUES et al., 2012; ROMERO et al., 2012; STEIDLE NETO et al., 2014). Nestes estudos, o desempenho do sistema no controle da duração e da frequência de irrigação é avaliado a partir da quantificação de indicadores de crescimento e de produtividade ao longo do ciclo da cultura. 
O presente trabalho teve por objetivo identificar o efeito de diferentes níveis de estresse hídrico no crescimento das cultivares RB92579, RB855453, RB867515 e RB928064, a partir da implementação de um sistema automático para o controle dos eventos de fertirrigação, durante o período de formação da cana-de-açúcar.

\section{MATERIAL E MÉTODOS}

\section{Local e técnicas de cultivo da cana-de-açúcar}

O experimento foi conduzido em uma casa de vegetação não climatizada, localizada na área experimental do Programa de Pós-Graduação em Meteorologia Agrícola, pertencente ao Departamento de Engenharia Agrícola (DEA), da Universidade Federal de Viçosa (UFV), Viçosa -MG.

No dia 17-12-2011, toletes das cultivares RB92579, RB855453, RB867515 e RB928064 foram plantados em vasos plásticos, sendo que estes foram preenchidos com $10 \mathrm{~L}$ de um substrato comercial (Bioplant, Nova Ponte-MG) à base de casca de Pínus, agentes agregantes, vermiculita, fibra de coco e complementos minerais (NPK e micronutrientes). No momento do plantio, dois toletes foram colocados em cada vaso para reduzir possíveis falhas de germinação. Após a emergência, realizou-se um desbaste para que apenas uma planta fosse mantida em cada recipiente. Nessa etapa, buscou-se eliminar as plantas com características morfológicas distintas, visando à obtenção de um padrão homo gêneo de crescimento vegetal.

Em decorrência da uniformidade das condições de cultivo, o experimento foi conduzido em delineamento inteiramente casualizado, com 16 tratamentos e cinco repetições, totalizando 80 unidades experimentais. Para a utilização total do espaço interior da casa de vegetação, foram cultivadas 192 plantas de cana-de-açúcar, sendo que, no entanto, foram utilizadas 80 plantas nas análises de crescimento, as quais foram avaliadas como unidades experimentais. As amostragens foram não destrutivas e realizadas semanalmente para a quantificação do crescimento.

Os tratamentos foram dispostos em esquema fatorial 4 x 4 (quatro cultivares e quatro níveis de estresse hídrico). As quatro cultivares foram submetidas a períodos alternados de estresse e de reposição hídrica no substrato, por meio de fertirrigações periódicas, cujos limites do potencial matricial de água $(\Psi)$ estiveram compreendidos entre a capacidade de campo e os valores de -10 (ausência de estresse), -60 (estresse leve), -90 (estresse moderado) e $-120 \mathrm{kPa}$ (estresse severo). As denominações "ausência de estresse" e "estresses leve, moderado e severo" foram aqui utilizadas somente para propósitos comparativos, uma vez que as plantas também são afetadas pelo tempo de exposição à deficiência de água e não somente pela sua intensidade. Neste experimento, os tratamentos com estresse hídrico foram iniciados aos 60 dias após o plantio.

Durante o período experimental, foram realizadas fertirrigações periódicas para o fornecimento complementar de nutrientes para as plantas. A solução nutritiva foi preparada manualmente em um reservatório (Figura 1A), onde foi utilizado um fertilizante comercial denominado "Peters Professional" (Peters Professional 20-20-20 GP, Marysville, OH, USA). Este fertilizante possuía a seguinte composição: $20 \%$ de $\mathrm{N} ; 20 \%$ de $\mathrm{P}_{2} \mathrm{O}_{5} ; 20 \%$ de $\mathrm{K}_{2} \mathrm{O} ; 0,05 \%$ de $\mathrm{Mg}$; $0,0068 \%$ de B; $0,0036 \%$ de $\mathrm{Cu} ; 0,05 \%$ de Fe; $0,025 \%$ de $\mathrm{Mn} ; 0,0009 \%$ de Mo e $0,0025 \%$ de Zn. 

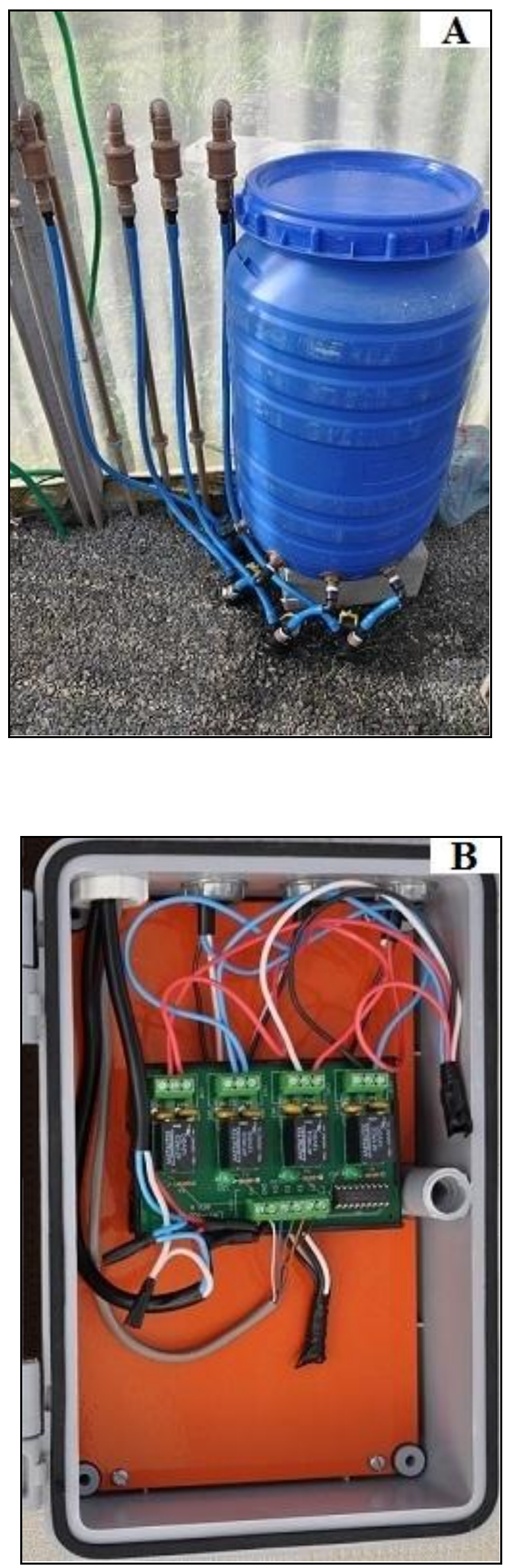

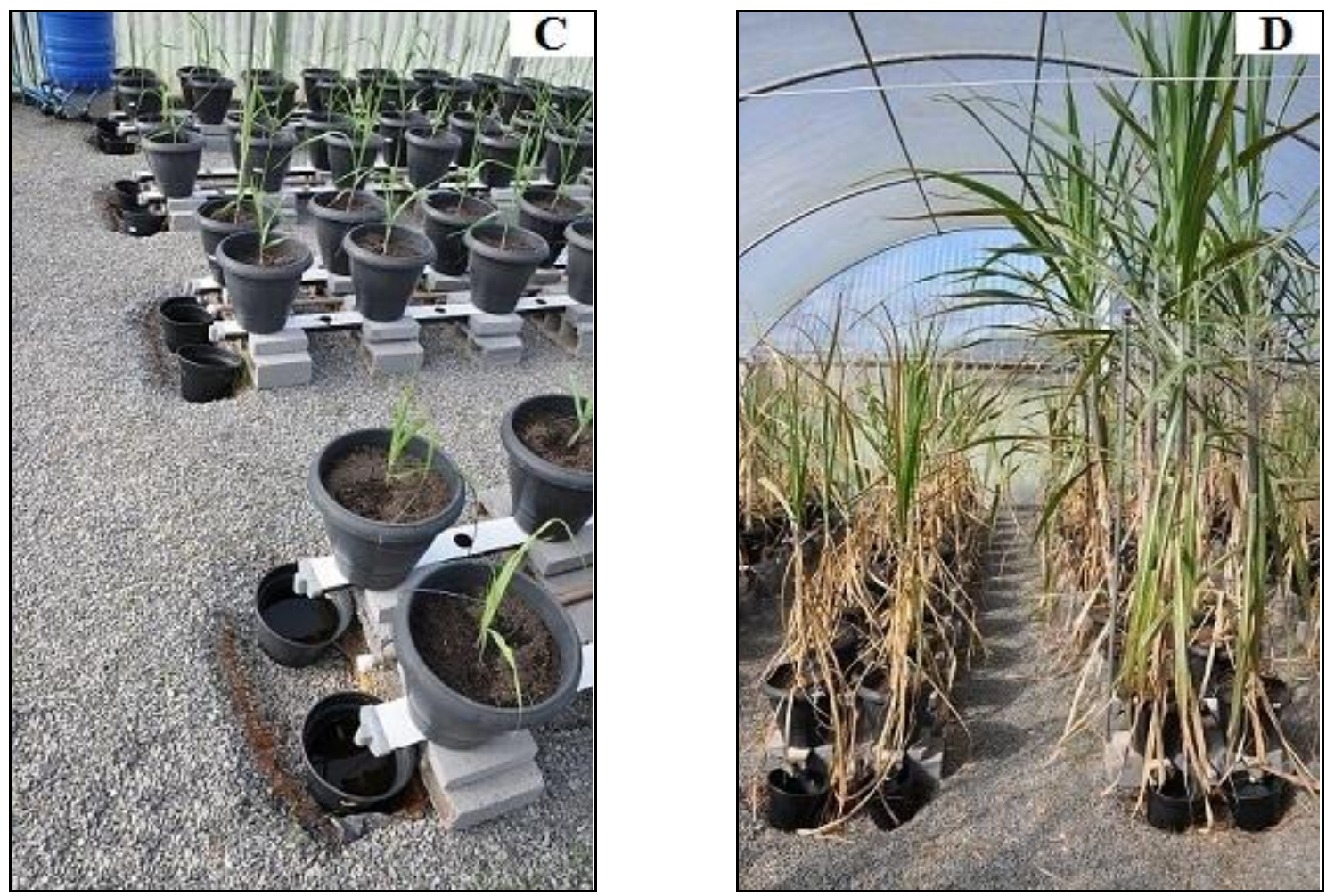

FIGURA 1. Sistema automático de fertirrigação para a avaliação do efeito do estresse hídrico em cultivares de cana-de-açúcar, o qual consiste em um reservatório para armazenamento da solução nutritiva (A); relés de controle dos eventos de fertirrigação (B); captação da solução nutritiva drenada (C), e plantas de cana-de-açúcar sob distintos tratamentos de estresse hídrico (D). Automatic fertigation system for evaluation of the water stress effect on sugarcane cultivars, which consisted of a nutrient solution reservoir (A), control relays of the fertigation events $(B)$, catchment of the drained nutrient solution $(C)$ and sugarcane plants under distinct water stress treatments (D).

As plantas foram fertirrigadas por meio de microtubos flexíveis derivados de tubulações de PVC. A duração e a frequência dos eventos de fertirrigação foram determinadas por um microcomputador associado a uma placa de relés (Figura 1B), que realizou o acionamento do sistema com base nos sinais elétricos de sensores de tensão de água instalados no substrato de cultivo. As plantas foram tutoradas individualmente, para garantir o crescimento vertical e evitar o tombamento causado pelo próprio peso.

No fundo de cada vaso, foi feito um orifício circular, onde foi colocado um disco de tela plástica com malha de $1 \mathrm{~mm}$ e, acima deste, $500 \mathrm{~mL}$ de carvão vegetal. Este procedimento foi adotado para facilitar a drenagem do excesso de água através do substrato, proveniente das fertirrigações. A condutividade elétrica (CE) da solução percolada nos vasos foi monitorada por meio de um condutivímetro (modelo CDH-42, Omega, Stamford, CT, USA) e foi mantida entre 2,0 e 3,0 $\mathrm{dS} \mathrm{m} \mathrm{m}^{-1}$.

Um perfil hidropônico de polipropileno (Hidrogood, Taboão da Serra-SP) com 2,6 m de comprimento foi mantido a uma inclinação de $4 \%$ para a coleta da solução nutritiva drenada em cada fileira. $\mathrm{Na}$ extremidade mais baixa do perfil, foram instalados drenos que estavam conectados a um vaso de 4L, onde foi armazenada a solução excedente após a realização de cada evento de fertirrigação (Figura 1C). Cada perfil coletou a drenagem proveniente de seis vasos de cultivo, sendo então utilizado um total de 32 perfis de coleta para os 192 vasos cultivados com cana-de-açúcar. Durante o período de crescimento, os perfilhos foram removidos periodicamente, de forma a manter apenas uma planta em cada vaso (Figura 1D). 


\section{Sistema de aquisição de dados e de controle dos eventos de fertirrigação}

A temperatura e a umidade relativa do ar foram medidas por meio de um sensor conjugado (modelo HMP60, Vaisala, Woburn-MA, USA), que foi instalado no interior de um tubo de PVC aspirado. O objetivo do abrigo foi impedir a incidência direta da radiação solar sobre os elementos sensíveis do sensor e, concomitantemente, garantir a taxa de ventilação de $5 \mathrm{~m} \mathrm{~s}^{-1}$ (ALLEN et al., 1998). Para medida da radiação solar global, utilizou-se um piranômetro (modelo LI-200SA, LiCor, Lincoln-NE, USA). A velocidade do ar foi medida por um anemômetro de fio quente (modelo FMA-903-I, Omega, Stamford-CT, USA). O piranômetro e o anemômetro também foram instalados na torre de aspiração constituída de PVC. Inicialmente, os sensores foram instalados a 2,0 m da superfície do solo e, posteriormente, deslocados verticalmente para cima à medida que ocorreu o crescimento da cultura, procurando sempre mantê-los acima do dossel vegetativo.

Para a medição da tensão de água no substrato, foram utilizados quatro sensores Watermark (200-SS, Irrometer Company, Riverside, CA, USA) instalados a uma profundidade de $20 \mathrm{~cm}$. Este sensor baseia-se no método ind ireto para a determinação instantânea da umidade do solo, por meio da medida da resistência à passagem de corrente elétrica. Paralelamente, a temperatura do substrato foi obtida por meio de termopares de cobre-constantan (Tipo T, Omega, Stamford-CT, USA) e foi utilizada para a correção dos valores de resistência, necessários para a obtenção da tensão de água no substrato.

O monitoramento da tensão de água no substrato de cultivo e dos elementos meteorológicos no interior da casa de vegetação foi realizado por meio de um sistema computadorizado, onde foi desenvolvido um programa em linguagem $\mathrm{C}^{++}$Builder (Borland), que, além de possibilitar a aquisição de dados, foi responsável pelo acionamento das motobombas de fetirrigação quando os níveis críticos de tensão no substrato eram alcançados. O sistema de aquisição de dados foi composto por um módulo analógico (modelo LR-7018, LR Informática Industrial, Porto Alegre-RS), enquanto o acionamento das motobombas de fertirrigação foi realizado por relés que estavam conectados ao microcomputador.

Os dados de cada elemento meteorológico foram armazenados em um microcomputador, em períodos de 1 minuto, correspondentes à média de cinco medições realizadas em intervalos de $1 \mathrm{~s}$, no período compreendido entre 55 e 59 s. O número de eventos de fertirrigação em cada tratamento de estresse hídrico também foi armazenado pelo sistema de aquisição de dados.

\section{Avaliações biométricas e estatísticas}

Embora a imposição dos tratamentos com estresse hídrico tenha sido iniciada aos 60 dias após o plantio, o monitoramento biométrico das plantas teve início no dia 17-01-2012, caracterizando 30 dias após o plantio (DAP), enquanto o término ocorreu no dia 22-05-2012 (157 DAPs). As avaliações foram realizadas semanalmente, totalizando 19 amostragens ao longo do período experimental, nas quais foi obtida a estatura dos colmos, com o auxílio de uma régua graduada, a partir da superfície do substrato até a folha +1 .

Os valores medidos de estatura dos colmos $(\mathrm{EC})$ foram submetidos à análise de regressão não linear, tendo como variável independente graus-dia acumulados após o plantio, obtendo-se o ajuste do modelo sigmoidal com três parâmetros aos valores medidos, conforme demonstrado na [eq. (1)].

$$
\mathrm{EC}=\frac{\mathrm{a}}{\left(1+\exp \left(-\left(\mathrm{GD}-\mathrm{GD}_{0}\right) / \mathrm{b}\right)\right)}
$$

em que,

EC - estatura dos colmos, cm;

GD - graus-dia acumulados após o plantio, ${ }^{\circ} \mathrm{Cd}$

a, b e $\mathrm{GD}_{0}$ - parâmetros do modelo sigmoidal. 
A taxa de elongação dos colmos (TEC, $\mathrm{cm}^{\circ} \mathrm{Cd}^{-1}$ ), conhecida como um índice morfofisiológico, foi obtida pela derivação do modelo sigmoidal com três parâmetros, como sugerido por SILVA et al. (2009).

\section{RESULTADOS E DISCUSSÃO}

\section{Ele mentos meteorológicos}

Os maiores valores de radiação solar global $\left(\mathrm{R}_{\mathrm{g}}\right)$ foram observados nos primeiros meses do período experimental, que teve início no mês de dezembro de 2011. Até meados de abril de 2012, ocorreram valores em torno de $22 \mathrm{MJ} \mathrm{m}^{-2} \mathrm{~d}^{-1}$. Nesse período, a temperatura do ar média diária foi de $24,3{ }^{\circ} \mathrm{C}$, com extremos de 20,0 e $27,3{ }^{\circ} \mathrm{C}$. Estes valores de temperatura, combinados com os de umidade relativa do ar, resultaram em déficit de pressão de vapor d'água no ar ( $\mathrm{DPV}$ ar) de até 12,41 $\mathrm{hPa}$, o qual foi observado no mês de janeiro.

A partir do mês de abril de 2012, a temperatura do ar começou a diminuir gradativamente até o final do experimento, quando o valor médio foi $18,9{ }^{\circ} \mathrm{C}$. No que se refere à velocidade do ar, foram constatados valores médios da ordem de $0,17 \mathrm{~m} \mathrm{~s}^{-1}$.

\section{Estatura e taxa de elongação dos colmos}

O conhecimento do padrão de elongação dos colmos é de grande importância, devido à sua correlação positiva com a produtividade da cultura (CARLIN et al., 2008). Na Figura 2, é mostrada a variação da estatura dos colmos da cana-de-açúcar, em função dos graus-dia acumulados após o plantio, para as cultivares RB92579, RB855453, RB867515 e RB928064, sendo também apresentados os números correspondentes dos eventos de fertirrigação. Durante esse período, as plantas foram submetidas aos distintos níveis de estresse hídrico.

O modelo sigmoidal com três parâmetros foi ajustado aos valores observados de estatura dos colmos, sendo que os parâmetros, correspondentes aos tratamentos com diferentes disponibilidades hídricas, estão apresentados na Tabela 1. Com base na Equação 1 e nos parâmetros pertinentes, determinados pela análise estatística, foram obtidas as repectivas curvas das taxas de elongação dos colmos (TEC), para as quatro cultivares e quatro condições de diponibilidade hídrica, como mostrado na Figura 3.
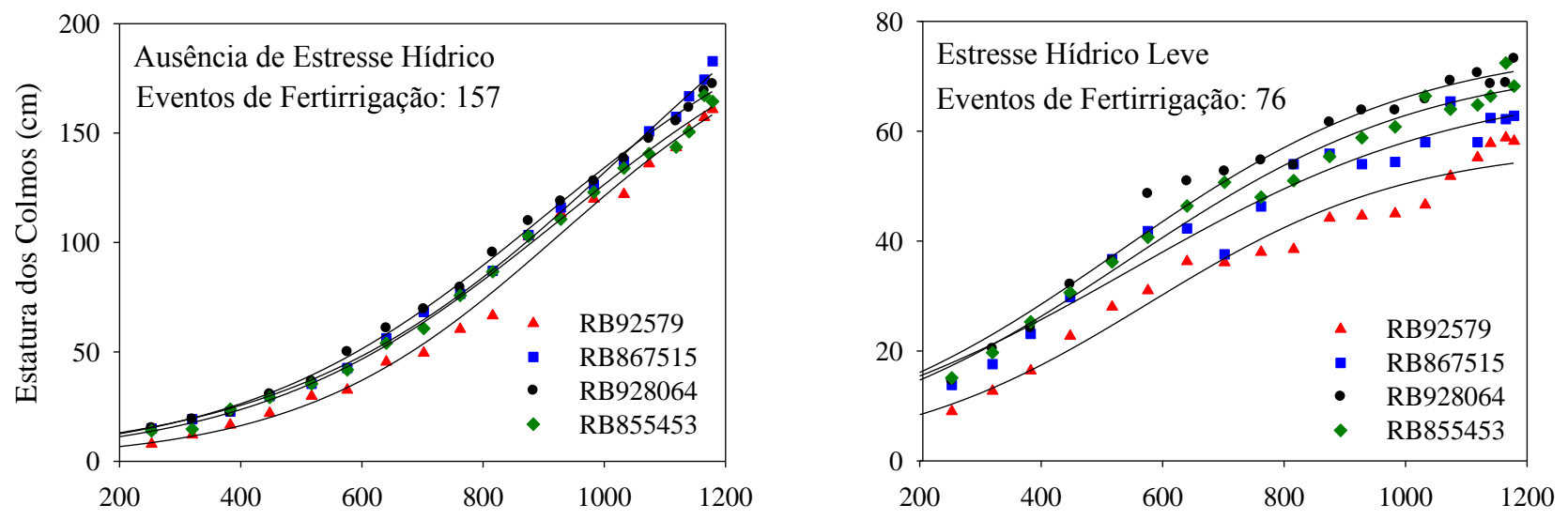

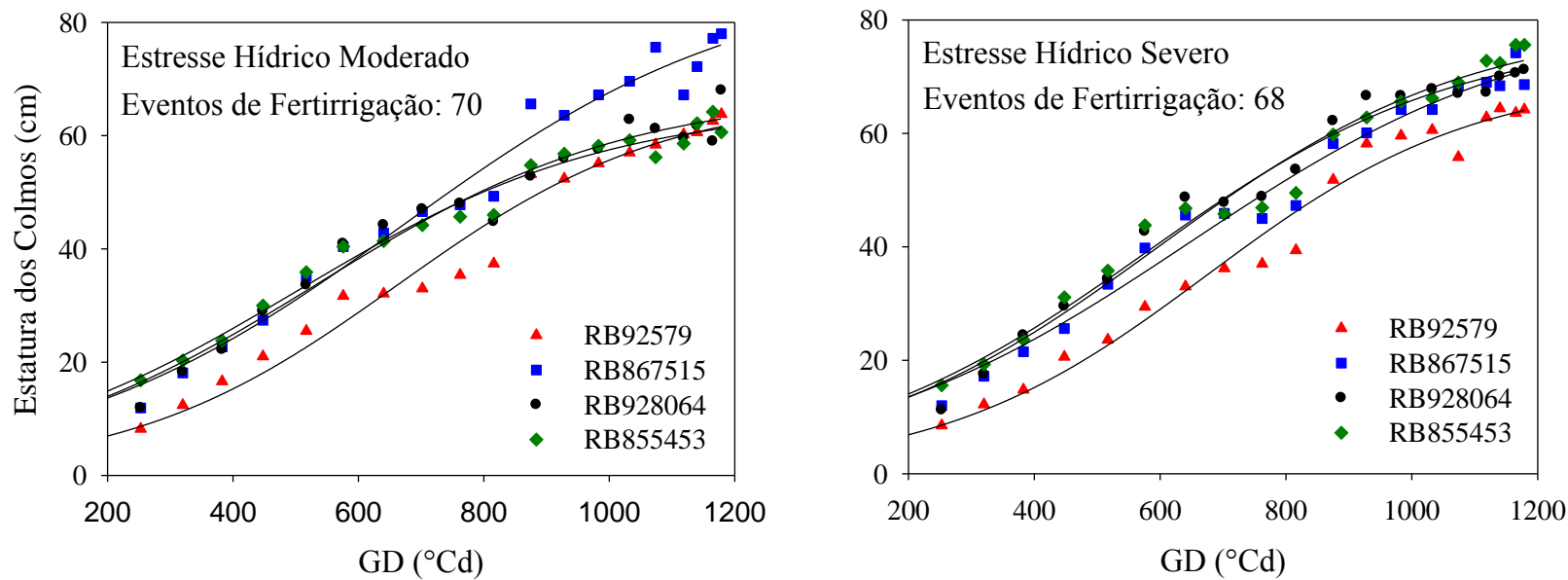

FIGURA 2. Variação da estatura dos colmos da cana-de-açúcar para as cultivares RB92579, RB855453, RB867515 e RB928064, submetidas aos tratamentos com ausência de estresse hídrico, estresses leve, moderado e severo, em função dos graus-dia acumulados após o plantio, com os respectivos eventos de fertirrigação. Sugarcane stalk lenght variation for cultivars RB92579, RB855453, RB867515 and RB928064, subjected to absence of water stress and mild, moderate and severe water stress, as a function of accumulated degree-days after planting, with the respective fertigation events.

OLIVEIRA et al. (2010) relataram a utização de um modelo sigmoidal similar ao do presente trabalho para caracterizar a variação da altura das plantas, ao a valiarem o crescimento da parte aérea de cultivares de cana-de-açúcar no Estado de Pernambuco. Os autores observaram três fases de desenvolvimento distintas para esta variável, onde, na primeira, ocorrida do plantio aos 60 DAPs, o crescimento foi lento e os valores médios observados foram de $18 \mathrm{~cm}$. Na segunda fase, dos 60 aos 240 DAPs, o desenvolvimento da planta foi rápido, com elevadas taxas de crescimento $\left(22 \mathrm{mmd}^{-1}\right)$. Já na terceira fase, as cultivares apresentaram ganhos médios de $49 \mathrm{~cm}$, o que representou $15 \%$ da altura total das plantas. 
TABELA 1. Parâmetros estimados pelo modelo sigmoidal ( $a, b$ e $x_{0}$ ) e coeficientes de determinação ajustados $\left(\mathrm{R}^{2}\right.$ aj $)$ para a estatura dos colmos de cultivares de cana-deaçúcar, em função dos graus-dia acumulados após o plantio. Estimated parameters by the sigmoid model $\left(a, b\right.$ and $\left.x_{0}\right)$ and adjusted determination coefficients $\left(R^{2}{ }_{\text {aj }}\right)$ for stalk length of sugarcane cultivars, as a function of accumulated degree-days after planting.

\begin{tabular}{|c|c|c|c|c|c|}
\hline & \multicolumn{4}{|c|}{ Parâmetros do Modelo Sigmoidal } \\
\hline & & $\mathrm{a}$ & $\mathrm{b}$ & $\mathrm{x}_{0}$ & $\mathrm{R}_{\text {aj }}^{2}$ \\
\hline \multirow{4}{*}{$\begin{array}{c}\text { Ausência de } \\
\text { estresse }\end{array}$} & RB92579 & $206,9 \pm(17,3)$ & $213,9 \pm(18,8)$ & $925,9 \pm(43,1)$ & 0,9901 \\
\hline & RB867515 & $285,4 \pm(18,4)$ & $276,9 \pm(11,8)$ & $1041,7 \pm(37,6)$ & 0,9980 \\
\hline & RB928064 & $224,5 \pm(8,3)$ & $248,5 \pm(9,5)$ & $901,7 \pm(21,9)$ & 0,9980 \\
\hline & RB855453 & $221,2 \pm(12,8)$ & $248,8 \pm(13,8)$ & $927,9 \pm(33,5)$ & 0,9961 \\
\hline \multirow{4}{*}{ Estresse leve } & RB92579 & $57,4 \pm(1,62)$ & - & $577,6 \pm(25,4)$ & 0,9530 \\
\hline & RB867515 & $69,3 \pm(1,98)$ & - & $545,9 \pm(29,8)$ & 0,9546 \\
\hline & RB928064 & $76,0 \pm(1,46)$ & - & $527,0 \pm(19,7)$ & 0,9753 \\
\hline & RB855453 & $72,9 \pm(1,28)$ & - & $542,3 \pm(17,6)$ & 0,9800 \\
\hline \multirow{4}{*}{$\begin{array}{l}\text { Estresse } \\
\text { moderado }\end{array}$} & RB92579 & $67,1 \pm(1,85)$ & - & $661,2 \pm(22,3)$ & 0,9693 \\
\hline & RB867515 & $88,1 \pm(2,52)$ & $\longrightarrow$ & $669,3 \pm(25,5)$ & 0,9742 \\
\hline & RB928064 & $67,7 \pm(1,57)$ & - & $534,9 \pm(23,5)$ & 0,9653 \\
\hline & RB855453 & $65,3 \pm(1,07)$ & & $503,4 \pm(17,3)$ & 0,9787 \\
\hline \multirow{4}{*}{$\begin{array}{c}\text { Estresse } \\
\text { severo }\end{array}$} & RB92579 & $70,1 \pm(1,73)$ & - & $675,0 \pm(19,7)$ & 0,9774 \\
\hline & RB867515 & $81,5 \pm(2,07)$ & - & $646,7 \pm(23,3)$ & 0,9772 \\
\hline & RB928064 & $77,7 \pm(1,55)$ & - & $575,4 \pm(19,2)$ & 0,9790 \\
\hline & RB855453 & $79,9 \pm(1,80)$ & - & $596,4 \pm(21,1)$ & 0,9740 \\
\hline
\end{tabular}

NOTA: 1) Os parâmetros do modelo sigmoidal são significativos ao nível de $1 \%$ de probabilidade, pelo teste t.

2) Os valores entre parênteses correspondem ao erro-padrão de estimativa dos parâmetros.

3) O parâmetro “ "b" está relacionado com a taxa máxima de elon gação relativa do colmo, que ocorre após a emer gência. Assim, para os tratamentos com estresses hídricos leve, moderado e severo, iniciados 60 dias após o plantio, este parâmetro foi estimado, para as respectivas cultivares, pelos valores apresentados nas linhas iniciais da presente tabela.

Os maiores valores de estatura dos colmos para as quatro cultivares avaliadas ocorreram no tratamento sem estresse hídrico, como ilustrado na Figura 2. Com base nos resultados obtidos ao final do experimento (157 DAP), os valores médios observados dessa variável foram, em ordem

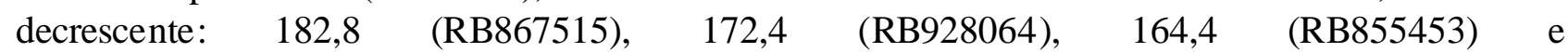
$160,8 \mathrm{~cm}$ (RB92579), tendo as duas primeiras cultivares apresentado diferenças significativamente maiores que as duas últimas (Teste de Tukey, a 5\% de probabilidade). 

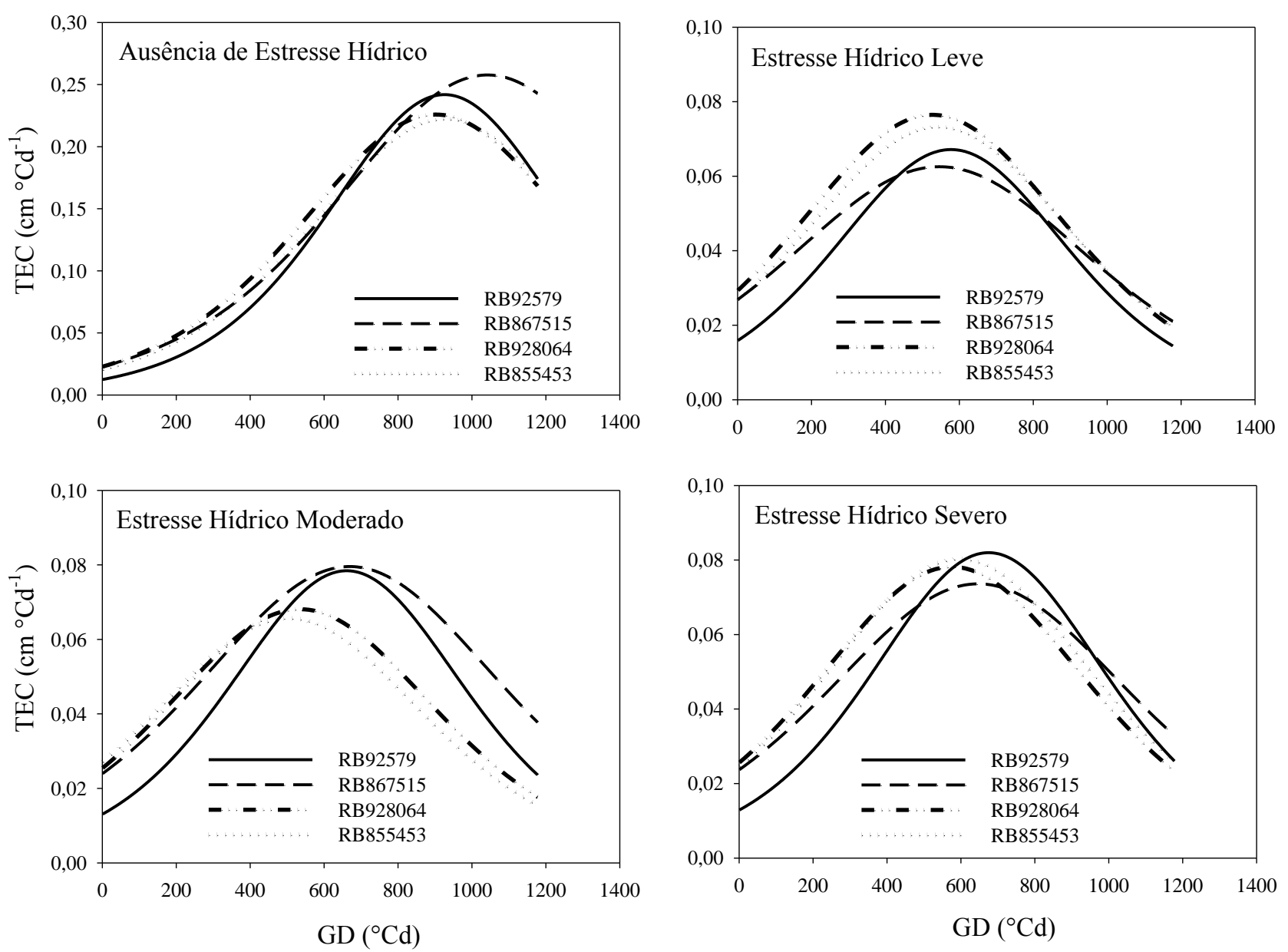

FIGURA 3. Taxa de elongação dos colmos (TEC) de cultivares de cana-de-açúcar em função dos graus-dia acumulados após o plantio (GD), para distintos níveis de estresse hídrico. Stem elongation rate (TEC) of sugarcane cultivars as a function of accumulated degree-days after planting (GD), for distinct wate r stress levels.

Foi possível verificar duas fases de desenvolvimento, sendo a primeira do plantio até $550^{\circ} \mathrm{Cd}$ (60 DAPs), caracterizada por crescimento lento, com valores da ordem de 29,0 (RB928064), 27,5 (RB867515), 26,5 (RB855453) e 20,0 cm (RB92579). Nessa fase, as cultivares apresentaram baixos valores de taxa de elongação do colmo (TEC) (Figura 3: Sem Estresse Hídrico), com valores médios próximos a $0,065 \mathrm{~cm}^{\circ} \mathrm{Cd}^{-1}$ para as cultivares $\mathrm{RB} 867516$, RB928064 e RB855453 e 0,051 $\mathrm{cm}^{\circ} \mathrm{Cd}^{-1}$ para a cultivar RB92579.

A segunda fase de desenvolvimento, ocorrida a partir de $550{ }^{\circ} \mathrm{Cd}$ (60 DAPs) foi caracterizada por crescimento rápido e linear. Os valores máximos da TEC observados nesse tratamento foram da ordem de $0,23 \mathrm{~cm}^{\circ} \mathrm{Cd}^{-1}$ para as cultivares RB92579, RB928064 e RB855453, sendo constatada por volta dos $920{ }^{\circ} \mathrm{Cd}$, enquanto, para a cultivar RB867515, os valores máximos de TEC foram da ordem de 0,26 $\mathrm{cm}^{\circ} \mathrm{Cd}^{-1}$ e ocorreram mais tarde $\left(1040{ }^{\circ} \mathrm{Cd}\right)$, explicando assim sua maior estatura aos 157 DAPs.

Segundo RAMESH (2000), o crescimento da cultura em estatura é favorecido em condições de alta temperatura e disponibilidade de água. Portanto, o crescimento contínuo, caracterizado por altas taxas de elongação do colmo observadas no tratamento com ausência de estresse, pode ser explicado pelos elevados valores de temperatura no interior da casa de vegetação e pelas condições plenas de suprimento hídrico, sob as quais as cultivares foram submetidas ao longo do período experimental.

Nos demais tratamentos, denominados de estresse hídrico leve, moderado e severo (Figura 2), o crescimento das plantas foi negativamente afetado. Os valores médios observados aos 
157 DAPs estiveram compreendidos entre 58,2 (RB92579) e 78,0 (RB928064), 60,6 (RB855453) e 78,0 (RB867515) e entre 64,2 (RB92579) e 75,6 cm (RB855453), para os tratamentos referidos como estresses leve, moderado e severo, respectivamente. Em geral, o estresse hídrico causou reduções na estatura dos colmos de aproximadamente $60 \%$ em comparação ao tratamento sem estresse hídrico.

Os efeitos negativos do estresse hídrico na estatura de plantas de cana-de-açúcar foram relatados por SILVA et al. (2008), ao estudarem os componentes do rendimento de genótipos de cana-de-açúcar para a região do Texas, Estados Unidos. Os autores verificaram que a estatura média dos colmos diminuiu de 192,69 para 167,48 cm, sob as condições de ausência de estresse hídrico e estresse hídrico moderado, respectivamente. Os mesmos autores enfatizam a importância da seleção de cultivares que se revelem capazes de manter a elongação dos colmos sob as condições de estresse hídrico.

A Figura 4 ilustra as reduções da estatura dos colmos causadas pelo estresse hídrico, apenas para três plantas de cada cultivar. Observou-se que a deficiência hídrica promoveu reduções drásticas no tamanho e no número de entrenós. MACHADO et al. (2009), estudando os efeitos da deficiência hídrica na biometria de plantas de cana-de-açúcar, nas fases de crescimento inicial, máximo crescimento e de acúmulo de sacarose, verificaram que o comprimento dos entrenós foi menos afetado à medida que o estresse hídrico foi retardado.

Com base nas curvas obtidas pela derivação do modelo sigmoidal, em relação aos graus-dia acumulados após o plantio, observa-se que as reduções da TEC, demonstradas pelas cultivares submetidas aos tratamentos com estresse hídrico, foram superiores a $65 \%$ em comparação à TEC das cultivares no tratamento com ausência de estresse.

No tratamento com estresse leve, os valores máximos da TEC, em ordem decrescente, foram 0,0765 (RB928064), 0,0732 (RB855453), 0,0672 (RB92579) e 0,0625 $\mathrm{cm}^{\circ} \mathrm{Cd}^{-1}$ (RB867515). Além disso, no tratamento especificado como estresse moderado, nota-se que a cultivar RB92579 apresentou os menores valores iniciais da TEC. Entretanto, esta cultivar possui valores máximos da TEC superiores aos da RB855453 e RB928064 para este tratamento. As cultivares RB92579 e RB867515 destacaram-se em relação às demais nas condições de estresse hídrico impostas pelo tratamento moderado, onde se verificou a TEC crescente até por volta dos $680{ }^{\circ} \mathrm{Cd}$. Por outro lado, as cultivares RB855453 e RB928064 mostraram diminuições logo aos $530{ }^{\circ} \mathrm{Cd}$. Ao final do período experimental, a cultivar RB867515 apresentou a maior TEC $\left(0,0377 \mathrm{~cm}^{\circ} \mathrm{Cd}^{-1}\right)$, seguida pela RB92579 $\left(0,0236 \mathrm{~cm}^{\circ} \mathrm{Cd}^{-1}\right), \operatorname{RB} 928064\left(0,0177 \mathrm{~cm}^{\circ} \mathrm{Cd}^{-1}\right)$ e $\operatorname{RB} 855453\left(0,0153 \mathrm{~cm}^{\circ} \mathrm{Cd}^{-1}\right)$.

Similarmente ao observado nos tratamentos com estresses leve e moderado, as plantas submetidas ao tratamento com estresse severo revelaram acentuadas quedas na TEC. Mais uma vez, as cultivares RB92579 e RB867515 mostraram reduções mais tardias, cujos valores máximos foram de 0,082 e $0,073 \mathrm{~cm}^{\circ} \mathrm{Cd}^{-1}$, respectivamente, ocorridos aos $660{ }^{\circ} \mathrm{Cd}$. A TEC máxima das cultivares RB928064 e RB855453 foi da ordem de $0,079 \mathrm{~cm}^{\circ} \mathrm{Cd}^{-1}$, observada por volta dos $580{ }^{\circ} \mathrm{Cd}$. 

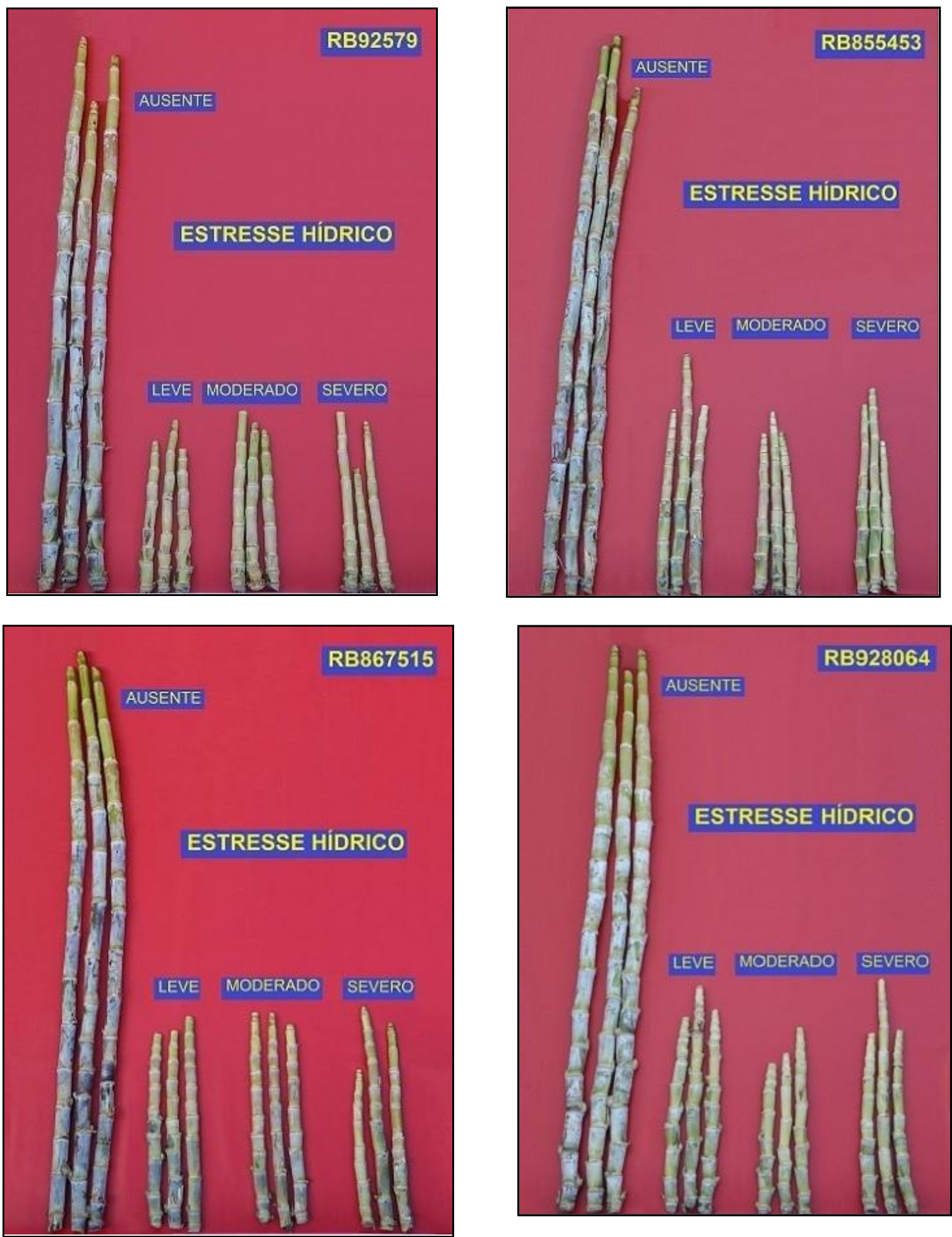

FIGURA 4. Ilustração do efeito do estresse hídrico na estatura dos colmos da cana-de-açúcar para as cultivares RB92579, RB855453, RB867515 e RB928064. As plantas foram submetidas aos potenciais matriciais de água de -10 (estresse ausente), -60 (estresse leve), -90 (estresse moderado) e $-120 \mathrm{kPa}$ (estresse severo). Illustration of the water stress effect on the sugarcane stalk length for cultivars RB92579, RB855453, RB867515 and RB928064. The plants were subjected to matric water potential of -10 (stress absent), -60 (mild stress), -90 (mode rate stress) and $-120 \mathrm{kPa}$ (severe stress).

INMAN-BAMBER (2004), quando pesquisou os critérios para irrigação na cultura da canade-açúcar, observou que a taxa de elongação dos colmos variou de acordo com a disponibilidade de água. INMAN-BAMBER \& SMITH (2005), estudando as relações de água na cultura da cana- 
de-açúcar, verificaram que a taxa de elongação dos colmos foi altamente sensível ao estresse hídrico. Segundo os autores, este é um parâmetro que pode ser utilizado como critério no manejo da irrigação na cana-de-açúcar.

\section{CONCLUSÕES}

O modelo sigmoidal com três parâmetros, tendo graus-dia acumulados após o plantio como variável independente, pode ser utilizado em estudos de avaliação do efeito do estresse hídrico no período de formação da cana-de-açúcar, uma vez que apresentou desempenho satisfatório para estimar a estatura dos colmos das cultivares RB92579, RB855453, RB867515 e RB928064.

Períodos intermitentes de estresse hídrico, com valores do potencial matricial de água inferiores a $-60 \mathrm{kPa}$, classificado como estresse leve no presente trabalho, promovem reduções severas nas taxas de elongação dos colmos. Portanto, não há a necessidade de reduções mais expressivas do potencial de água para a avaliação da suscetibilidade ou de tolerância de cultivares de cana-de-açúcar ao estresse hídrico.

$\mathrm{Na}$ ausência de estresse hídrico, os maiores valores de estatura dos colmos ao final do período experimental foram, em ordem decrescente, observados nas cultivares RB867515, RB928064, RB855453 e RB92579. As cultivares RB92579 e RB867515 destacaram-se em relação às cultivares RB928064, RB855453 nas condições de estresse hídrico moderado, onde se verificou a taxa de elongação dos colmos crescente até por volta dos $680{ }^{\circ} \mathrm{Cd}$. Por outro lado, as cultivares RB855453 e RB928064 mostraram diminuições logo aos $530{ }^{\circ} \mathrm{Cd}$.

\section{AGRADECIMENTOS}

Esta pesquisa foi realizada com o apoio financeiro da Fundação de Amparo à Pesquisa do Estado de Minas Gerais (FAPEMIG) e do Conselho Nacional de Desenvolvimento Científico e Tecnológico $(\mathrm{CNPq})$. O primeiro autor também gostaria de agradecer à Universidade Federal de Viçosa, por meio do Programa de Pós-Graduação em Meteorologia Agrícola, pela oportunidade para a obtenção do título de Mestre.

\section{REFERÊNCIAS}

ALLEN, R.G.: PEREIRA, L.S.; RAES, D.; SMITH, M. Crop evapotranspiration: guidelines for computing crop requiriments. Roma: FAO, 1998. 328p. (Irrigation and drainage paper, 56).

BATISTA, E.L. da S.; ZOLNIER, S.; RIBEIRO, A.; LYRA G.B.; SILVA, T.G.F. da; BOEHRINGER, D. Modelagem do crescimento de cultivares de cana-de-açúcar no período de formação da cultura. Revista Brasileira de Engenharia Agrícola e Ambiental, Campina Grande, v.17, n.10, p.1080-1087, 2013.

BOEHRINGER, D.; ZOLNIER, S.; RIBEIRO, A.; STEIDLE NETO, A.J. Determinação do flux o de seiva na cana-de-açúcar pelo método do balanço de energia caulinar. Engenharia Agrícola, Jaboticabal, v.33, n.2, p.237-248, 2013.

CARLIN, S.D.; SILVA, M.A.; ROSSETO, R. Parâmetros biométricos e produtividade da cana-deaçúcar após tombamento dos colmos. Bragantia, Campinas, v.67, n.4, p.845-853, 2008.

CONAB - Companhia Nacional de Abastecimento. Acompanhamento da Safra Brasileira: Canade-açúcar: Safra 2013/2014 - terceiro levantamento, 2013. Disponível em:

http://www.conab.gov.br. Acesso em: 8 fev. 2014.

DOMINGUES, D.S.; TAKAHASHI, H.W.; CAMARA, C.A.P.; NIXDORF, S.L. Automated system developed to control $\mathrm{pH}$ and concentration of nutrient solution evaluated in hydroponic lettuce production. Computers and Electronics in Agriculture, New York, v.84, p.53-61, 2012.

GAVA, G.J. de C.; SILVA, M. de A.; SILVA, R.C. da; JERONIMO, E.M.; CRUZ, J.C.S.;

KÖLLN, O.T. Produtividade de três cultivares de cana-de-açúcar sob manejos de sequeiro e 
irrigado por gotejamento. Revista Brasileira de Engenharia Agrícola e Ambiental, Campina Grande, v.15, n.3, p.250-255, 2011.

GONÇALVES, E.R; FERREIRA, V.M.; SILVA, J.V.; ENDRES L.; BARBOSA, T.P; DUARTE, W. de G. Trocas gasosas e fluorescência da clorofila $a$ em variedades de cana-de-açúcar submetidas à deficiência hídrica. Revista Brasileira de Engenharia Agrícola e Ambiental, Campina Grande, v.14, n.4, p.378-386, 2010.

INMAN-BAMBER, N.G. Sugarcane water stress criteria for irrigation and drying off. Field Crops Research, Amsterdam, v.89, p.107-122, 2004.

INMAN-BAMBER N.G.; SMITH, D.M. Water relations in sugarcane and response to water deficits. Field Crops Research, Amsterdam, v.92, p.185-202, 2005.

LACLAU, P.B.; LACLAU, J.P. Growth of the whole root system for a plant crop of sugarcane under rainfed and irrigated environments in Brazil. Field Crops Research, Amsterdam, v.114, p.351-360, 2009.

LYRA G.B.; BATISTA, E.L.S.; LYRA, G.B.; PEREIRA, C.R.; SILVA, L.D.B.; SILVA, G.M. Coeficiente da cultura da cana-de-açúcar no estádio inicial de desenvolvimento em Campos dos Goytacazes, RJ. Irriga, Botucatu, v.17, n.1, p.102-113, 2012.

MACHADO, R.S.; RIBEIRO, R.V.; MARCHIORI, P.E.R.; MACHADO, D.F.S.P.; MACHADO, E.C.; LANDELL, M.G.A. Respostas biométric as e fisiológicas ao déficit hídrico em cana-de-açúcar em diferentes fases fenológicas. Pesquisa Agropecuária Brasileira, Brasília, v.44, n.12, p.1575$1582,2009$.

NOBILE, F.O. de; GALBIATTI, J.A.; MURAISHI, R.I.; ARAUJO, J.R. Variáveis biométricas da cana-de-açúcar fertilizada com resíduos orgânico e ind ustrial e irrigada com água servida e potável. Engenharia Agrícola, Jaboticabal, v.31, n.1, p.193-200, 2011.

OLIVEIRA, E.C.A. de; FREIRE, F.J.; OLIVEIRA, A.C. de; SIMÕES NETO, D.E.; ROCHA, A.T. da; CARVALHO, L.A. de. Produtividade, eficiência de uso da água e qualidade tecnológica de cana-de-açúcar submetida a diferentes regimes hídricos. Pesquisa Agropecuária Brasileira, Brasília, v.46, n.6, p.617-625, 2011.

OLIVEIRA, R. A.; DAROS, E.; ZAMBON, J. L. C.; WEBER, H.; IDO, O. T.; ZUFFELLATORIBAS, K. C.; KOEHLER, H. S.; SILVA, D. K. T. Crescimento e desenvolvimento de três cultivares de cana-de-açúcar, em cana-planta, no Estado do Paraná: taxas de crescimento. Scientia Agraria, Curitiba, v.6, n.1-2, p.85-89, 2005.

OLIVEIRA, E.C.A.; OLIVEIRA, R.I.; ANDRADE, B.M.T.; FREIRE, F.J.; JÚNIOR, M.A.L.; MACHADO, P.R. Crescimento e acúmulo de matéria seca em variedades de cana-de-açúcar cultivadas sob irrigação plena. Revista Brasileira de Engenharia Agrícola e Ambiental, Campina Grande, v.14, n.9, p.951-960, 2010.

RAMESH, P. Effect of different levels of drought during the formative phase on growth parameters and its relationship with dry matter accumulation in sugarcane. Journal of Agronomy and Crop Science, Berlin, v.185, p.83-89, 2000.

ROMERO, R.; MURIEL, J.L.; GARCÍA, I.; PENÃ, D.M. de la. Research on automatic irrigation control: state of the art and recent results. Agricultural Water Management, Amsterdam, v.114, p.59-66, 2012.

SMITH, D.M.; INMAN-BAMBER, N.G.; THORBURN, P.J. Growth and function of the sugarcane root system. Field Crops Research, Amsterdam, v.92, p.169-183, 2005.

SILVA, M. de A.; SILVA, J.A.G. da; ENCISO, J.; SHARMA, V.; JIFON, J. Yield components as ind icators of drought tolerance of sugarcane. Scientia Agricola, Piracicaba, v.65, n.6, p.620-627, 2008. 
SILVA, T.G.F. da; ZOLNIER, S.; GROSSI, J.A.S.; BARBOSA, J.G.; MOURA, C.R.W.;

MUNIZ, M. A. Crescimento do giras sol ornamental cultivado em ambiente protegido sob diferentes níveis de condutividade elétrica de fertirrigação. Revista Ceres, Viçosa, MG, v.56, n.5, p.602-610, 2009.

SILVA, T.G.F. da; MOURA, M.S.B. de; ZOLNIER, S.; SOARES, J.M.; SOUZA, L.S.B. de; BRANDÃO, E.O. Variação do balanço de radiação e de energia da cana-de-açúcar irrigada no semiárido brasileiro. Revista Brasileira de Engenharia Agrícola e Ambiental, Campina Grande, v.15, n.2, p.139-147, 2011a.

SILVA, T.G.F. da; MOURA, M.S.B. de; ZOLNIER, S.; SOARES, J.M.; VIEIRA, V.J. de S.; GOMES JÚNIOR, W.F. Demanda hídrica e eficiência do uso de água da cana-de-açúcar irrigada no semiárido brasileiro. Revista Brasileira de Engenharia Agrícola e Ambiental, Campina Grande, v.15, n.12, p.1257-1265, 2011 b.

SILVA, V. de P.R. da; SILVA, B.B. da; ALBUQUERQUE, W.G.; BORGES, C.J.R.; SOUS A, I.F. de; DANTAS NETO, J. Crop coefficient, water requirements, yield and water use efficiency of sugarcane growth in Brazil. Agricultural Water Management, Amsterdam, v.128, p.102-109, 2013.

SILVA, T.G.F. da; MOURA, M.S.B. de; ZOLNIER, S.; CARMO, J.F.A. do; SOUZA, L.S.B. de; Biometria da parte aérea da cana soca irrigada no Submédio do Vale do São Francisco. Revista Ciência Agronômica, Fortaleza, v.43, n.3, p.500-509, 2012.

STEIDLE NETO, A.J.; ZOLNIER, S.; LOPES, D. de C. Development and evaluation of an automated system for fertigation control in soilless tomato production. Computers and Electronics in Agriculture, New York, v.103, p.17-25, 2014.

TRENTIN, R.; ZOLNIER, S.; RIBEIRO, A.; STEIDLE NETO, A.J. Transpiração e temperatura foliar da cana-de-açúcar sob diferentes valores de potencial matricial. Engenharia Agrícola, Jaboticabal, v.31, n.6, p.1085-1095, 2011.

ZHAO, D.; GLAZ, B.; COMSTOCK, J.C. Sugarcane response to water-deficit stress during early growth on organic and sand soils. American Journal of Agricultural and Biological Sciences, Vails Gate, v.5, p.403-414, 2010. 